\title{
Implications of the Higgs Boson Discovery for mSUGRA
}

\author{
Sujeet Akula, Pran Nath, and Gregory Peim \\ Department of Physics, Northeastern University, Boston, MA 02115, USA
}

\begin{abstract}
A Bayesian analysis is carried out to identify the consistent regions of the mSUGRA parameter space, where the newly-discovered Higgs boson's mass is used as a constraint, along with other experimental constraints. It is found that $m_{1 / 2}$ can lie in the sub-TeV region, $A_{0} / m_{0}$ is mostly confined to a narrow strip with $\left|A_{0} / m_{0}\right| \leq 1$, while $m_{0}$ is typically a TeV or larger. Further, the Bayesian analysis is used to set $95 \% \overline{C L}$ lower bounds on sparticle masses. Additionally, it is shown that the spin independent neutralino-proton cross section lies just beyond the reach of the current sensitivity but within the projected sensitivity of the SuperCDMS-1T and XENON-1T experiments, which explains why dark matter has thus far not been detected. The light sparticle spectrum relevant for the discovery of supersymmetry at the LHC are seen to be the gluino, the chargino and the stop with the gluino and the chargino as the most likely candidates.
\end{abstract}

Keywords: Higgs, LHC, Supersymmetry

Introduction: The most recent search at the LHC 1, 2, for the Higgs boson 3 with the combined $7 \mathrm{TeV}$ and $8 \mathrm{TeV}$ data indicates a signal for the Higgs boson with mass $125.3 \pm 0.4$ (stat.) \pm 0.5 (syst.) $\mathrm{GeV}$ for CMS with a local significance of $5.0 \sigma$ and with mass $126.0 \pm$ 0.4 (stat.) \pm 0.4 (syst.) $\mathrm{GeV}$ for ATLAS with local significance of $5.9 \sigma$. As is well known the Higgs boson mass at the tree level lies below the $Z^{0}$ boson mass, but it can be made larger by inclusion of loop corrections. However, in supergravity grand unification [4 there is another upper limit, i.e., of about $130 \mathrm{GeV}$ due to the constraint of radiative breaking of the electroweak symmetry (for a review see [5) as well as other experimental constraints (for a recent analysis see [6, 7]). The correction to the Higgs boson mass is given by [8] (for a review see [9])

$$
\Delta m_{h^{0}}^{2} \simeq \frac{3 m_{t}^{4}}{2 \pi^{2} v^{2}} \ln \frac{M_{\mathrm{S}}^{2}}{m_{t}^{2}}+\frac{3 m_{t}^{4}}{2 \pi^{2} v^{2}}\left(\frac{X_{t}^{2}}{M_{\mathrm{S}}^{2}}-\frac{X_{t}^{4}}{12 M_{\mathrm{S}}^{4}}\right)
$$

where $X_{t} \equiv A_{t}-\mu \cot \beta$, where $A_{t}$ is the $A_{0}$ parameter run down to the weak scale (see Eq. (2p), $v=246 \mathrm{GeV}$, and $M_{\mathrm{S}}$ is an average stop mass. The loop correction in Eq. (1) is maximized when $X_{t} \sim \sqrt{6} M_{S}$. There are also additional loop corrections from, e.g., the $b$-quark sector as well as from higher loops. The early searches at the LHC-7 gave a possible hint of the Higgs boson in the mass range $\sim(117-129) \mathrm{GeV}[10$ and the combined Tevatron analysis reported an excess between $(115-140) \mathrm{GeV}$ [11. These findings have led to significant activity [6, 12, 13] to investigate the implications of the results for supersymmetry.

Implications for mSUGRA: We note that the scale $M_{S}$ in Eq. (1) which is determined by the soft parameters depends sensitively on the Higgs mass. In the analysis we use the Higgs boson mass constraint within the Bayesian statistical framework to estimate the soft parameters of mSUGRA (sometimes referred to as CMSSM) which are given by [4]

$$
m_{0}, m_{1 / 2}, A_{0}, \tan \beta, \operatorname{sgn}(\mu)
$$

where $m_{0}$ is the universal scalar mass, $m_{1 / 2}$ is the universal gaugino mass, $A_{0}$ is the trilinear couplings and $\tan \beta$ is the ratio of the two Higgs VEVs in MSSM, and $\mu$ is the Higgs mixing parameter. The soft parameters of Eq. (1) define our model's parameter set, $\theta=$ $\left\{m_{0}, m_{1 / 2}, A_{0}, \tan \beta\right\}$, and additionally we consider a set of the most sensitive standard model nuisance parameters, $\psi=\left\{m_{t}, m_{b}\left(m_{b}\right)^{\overline{\mathrm{MS}}}, \alpha_{\mathrm{s}}\left(m_{Z}\right)^{\overline{\mathrm{MS}}}, \alpha_{\mathrm{EM}}\left(m_{Z}\right)^{\overline{\mathrm{MS}}}\right\}$. These together form the basis parameter set: $\Theta=\{\theta, \psi\}$. Using Bayes's theorem, the posterior probability density function (PDF) for the theory described by $\Theta$, which may be mapped to observables, $\xi(\Theta)$ to be compared against experimental data, $d$ is given by:

$$
p(\Theta \mid d)=\frac{p(d \mid \xi(\Theta)) \pi(\Theta)}{p(d)},
$$

where $\mathcal{L} \equiv p(d \mid \xi(\Theta))$ is the likelihood function-the terms of which are described in Table I $\pi(\Theta)$ is the distribution in $\Theta$ prior to considering experimental results, and $\mathcal{Z} \equiv p(d)$ is the Bayesian evidence which can be used in model selection. However, in our goal of parameter estimation, it serves only as a normalization factor. We present results obtained by considering both the 2D marginalized posterior PDF (where the full $\mathrm{N}$ dimensional posterior PDF of Eq. (3) has been integrated over the other parameters), as well as the profile likelihoods (where the confidence levels are determined by comparison to the global best-fit point). (For a more detailed description see the second reference in [18.)

The analysis was done by using SusyKIT 19, which employs the MultiNest [18] package for sampling parameter points efficiently, and uses SofTSUSY 20] for spectrum calculation, and MICROMEGAs 21] to calculate the relic density as well as for the indirect constraints. The credible intervals, marginalized posterior PDF's, and profile likelihood distributions were calculated using the plotting routines of SuperBayes [22, which is largely based on the tools provided by CosMoMC [23]. The constraint from the $g_{\mu}-2$ measure- 


\begin{tabular}{|c|c|c|c|c|c|}
\hline Observable & Central value & Exp. Err. & Th. Err. & Distribution & Ref. \\
\hline \multicolumn{6}{|c|}{ SM Nuisance Parameters } \\
\hline$m_{t}$ & $173.5 \mathrm{GeV}$ & $1.0 \mathrm{GeV}$ & - & Gaussian & {$[14]$} \\
\hline$m_{b}\left(m_{b}\right)^{\overline{\mathrm{MS}}}$ & $4.18 \mathrm{GeV}$ & $0.03 \mathrm{GeV}$ & - & Gaussian & 14 \\
\hline$\alpha_{\mathrm{S}}\left(m_{Z}\right)^{\overline{\mathrm{MS}}}$ & 0.1184 & $7 \times 10^{-4}$ & - & Gaussian & 14 \\
\hline $1 / \alpha_{\mathrm{EM}}\left(m_{Z}\right)^{\overline{\mathrm{MS}}}$ & 127.944 & 0.014 & - & Gaussian & 14 \\
\hline \multicolumn{6}{|c|}{ Measured } \\
\hline $\mathcal{B} r\left(\bar{B} \rightarrow X_{s} \gamma\right) \times 10^{4}$ & 3.21 & 0.33 & 0.21 & Gaussian & 16 \\
\hline$\Omega h^{2}$ & 0.1126 & 0.0036 & $10 \%$ & Upper-Gaussian & 15 \\
\hline \multirow{2}{*}{\multicolumn{6}{|c|}{ Limits (95\% CL) }} \\
\hline & & & & & \\
\hline $\mathcal{B} r\left(B_{s}^{0} \rightarrow \mu^{+} \mu^{-}\right)$ & $4.5 \times 10^{-9}$ & - & $14 \%$ & Upper - Error Fn & 17 \\
\hline$m_{h} 0$ & $122.5 \mathrm{GeV}$ & - & - & Lower - Step Fn & 10 \\
\hline$m_{h}^{0}$ & $129 \mathrm{GeV}$ & - & - & Upper - Step Fn & 10 \\
\hline$m_{\tilde{\chi}_{1}^{0}}^{n}$ & $46 \mathrm{GeV}$ & - & $5 \%$ & Lower - Error Fn & 14 \\
\hline$m_{\tilde{\chi}_{2}^{0}}^{0}$ & $62.4 \mathrm{GeV}$ & - & $5 \%$ & Lower - Error Fn & 14] \\
\hline$m_{\tilde{\chi}_{3}^{0}}^{2}$ & $99.9 \mathrm{GeV}$ & - & $5 \%$ & Lower - Error Fn & 14 \\
\hline$m_{\tilde{\chi}_{4}^{0}}^{0}$ & $116 \mathrm{GeV}$ & - & $5 \%$ & Lower - Error Fn & 14 \\
\hline${ }^{m} \tilde{\chi}_{1}^{ \pm}$ & $94 \mathrm{GeV}$ & - & $5 \%$ & Lower - Error Fn & 14 \\
\hline$m_{\tilde{e}_{R}}$ & $107 \mathrm{GeV}$ & - & $5 \%$ & Lower - Error Fn & 14 \\
\hline$m_{\tilde{\mu}_{R}}$ & $94 \mathrm{GeV}$ & - & $5 \%$ & Lower - Error Fn & 14 \\
\hline$m_{\tilde{\tau}_{1}}$ & $81.9 \mathrm{GeV}$ & - & $5 \%$ & Lower - Error Fn & 14 \\
\hline$m_{\tilde{b}_{1}}$ & $89 \mathrm{GeV}$ & - & $5 \%$ & Lower - Error Fn & 14 \\
\hline$m_{\tilde{t}_{1}}$ & $95.7 \mathrm{GeV}$ & - & $5 \%$ & Lower - Error Fn & 14 \\
\hline$m_{\tilde{g}}$ & $500 \mathrm{GeV}$ & - & $5 \%$ & Lower - Error Fn & 14 \\
\hline$m_{\tilde{q}}^{\mathcal{Y}}$ & $1100 \mathrm{GeV}$ & - & $5 \%$ & Lower - Error Fn & 14 \\
\hline
\end{tabular}

TABLE I: Summary of the observables used to estimate the mSUGRA parameters. Only the upper-half of the Gaussian is used in the consideration of $\Omega h^{2}$, i.e., there is only a penalty for values larger than the central value which allows for multicomponent dark matter 30. The 95\% CL limits have been evaluated under the assumption of only theoretical uncertainty, so the distribution used here is based on the error function, given explicitly in the fourth reference of $[22$.

ment is not imposed in this analysis and this issue will be discussed later in the text.

In our analysis, we took our prior knowledge of the parameters to be either flat linear distributions or flat logarithmic distributions, with $m_{0} \in(0.05,8) \mathrm{TeV}(\log )$, $m_{1 / 2} \in(0.05,5) \mathrm{TeV}(\log ), A_{0} \in(-30,30) \mathrm{TeV}$ (linear), and $\tan \beta \in(3,60)$ (linear). We have fixed $\operatorname{sgn}(\mu)$ to be positive. The Standard Model nuisance parameters were allowed to vary in $2 \sigma$ windows of their central values, as quoted in TableI Our MultiNest sampling parameters, as defined in [18], were $n_{\text {live }}=20,000$ and tol $=0.0001$. It has been shown in 24 and the second reference in 18 that these parameters are not only sufficient to provide a map of the posterior PDF, but also to find the true best-fit point which is essential for the profile likelihood analysis.

In our likelihood analysis we use the CMS result since that result was available earlier [1. We report our fits to the data, including the Higgs mass, in Fig. 1 in the form of 2D posterior PDF maps (left panels) as well as the profile likelihood maps (right panels). The posterior mean is marked with a large dot and the global best-fit is marked with a circled ' $\mathrm{X}$ '. (Note that while the bestfit point is crucial in Frequentist likelihood-ratio tests, it has no significance in the Bayesian framework.) The top panels exhibit the constraint in the $m_{0}-m_{1 / 2}$ plane and show that $m_{0}$ is typically a $\mathrm{TeV}$ or larger, while $m_{1 / 2}$ can lie below $500 \mathrm{GeV}$. The middle panels exhibit the constraint in the $A_{0} / m_{0}-\tan \beta$ plane, and here one finds that most of the allowed parameter space lies in the narrow strip $\left|A_{0} / m_{0}\right| \leq 1$ with a small strip in the range $\left|A_{0} / m_{0}\right| \in(-2,-6)$. The bottom panels exhibit the constraint in the $m_{A}-\tan \beta$ plane, and here one finds that the majority of the allowed range of $m_{A}$ lies above $1 \mathrm{TeV}$. Thus $m_{A} \gg m_{h^{0}}$ for the majority of the parameter space and thus we are in the so-called decoupling limit.

It was pointed out in [25] that most of the experimentally consistent parameter space of mSUGRA lies on the Hyperbolic Branch (HB) 26, 27] of radiative breaking of the electroweak symmetry under the LHC-7 constraints. The HB region has sub-regions which we may label as Focal Point (HB/FP), Focal Curves (HB/FCi, $\mathrm{i}=1,2)$, and Focal Surfaces (HB/FS). It was shown in 6, 25, that the $\mathrm{HB} / \mathrm{FP}$ is mostly depleted while the remaining parameter space lies on $\mathrm{HB} / \mathrm{FCi}$ or $\mathrm{HB} / \mathrm{FS}$. Specifically we note here that the right edge of $A_{0} / m_{0}$ in Fig. 1 is $\sim 1$. The value $\left|A_{0} / m_{0}\right|=1$ was argued as string-motivated in 28. and was shown to be the asymptotic limit on the focal curve $\mathrm{HB} / \mathrm{FC} 1$ in 25$]$.

In Fig. 2 we present the 2D posterior PDF's (left panels) and the profile likelihoods (right panels) in the planes of the phenomenologically important sparticle masses. The top panels present the results in the gluino-squark mass plane, and indicate that the gluino can be below a $\mathrm{TeV}$. The second row is plotted in the squark-chargino mass plane and demonstrates that the chargino masses are only bounded from below by the direct searches at LEP. The next row exhibits our fit in the stau-stop mass plane. Here one finds that the stau and stop masses are typically large except for a small strip where the stop mass can lie below a TeV. This is largely to be expected as we rely on a heavy stop to provide a sizable loop correction to the Higgs mass. The bottom panels show the analysis in the $\mu-m_{\tilde{g}}$ plane. One finds that $\mu$ is typically quite light, i.e., $\mu$ can be significantly below $500 \mathrm{GeV}$.

Using the marginalized $1 \mathrm{D}$ posterior $\mathrm{PDF}$ we are able to set lower limits on the sparticle masses from the $2 \sigma$ credible regions. We present those limits here: $m_{\tilde{g}}>$ $1.39 \mathrm{TeV}, m_{\tilde{\chi}_{1}^{ \pm}}>196 \mathrm{GeV}, m_{A_{0}} \sim m_{H_{0}} \sim m_{H^{ \pm}}>$ $1.3 \mathrm{TeV}, m_{\tilde{t}_{1}}>3.1 \mathrm{TeV}, m_{\tilde{\tau}_{1}}>3.1 \mathrm{TeV}, m_{\tilde{q}}>5 \mathrm{TeV}$, and $m_{\tilde{\ell}}>4.8 \mathrm{TeV}$. The profile likelihood analysis yields different results. Here, we find the $95 \%$ CL sparticle lower limits to be $m_{\tilde{g}}>690 \mathrm{GeV}, m_{\tilde{\chi}_{1}^{ \pm}}>95 \mathrm{GeV}$, $m_{A_{0} \sim H_{0} \sim H^{ \pm}}>540 \mathrm{GeV}, m_{\tilde{t}_{1}}>580 \mathrm{GeV}, m_{\tilde{\tau}_{1}}>$ $310 \mathrm{GeV}, m_{\tilde{q}}>1.5 \mathrm{TeV}$, and $m_{\tilde{\ell}}>580 \mathrm{GeV}$. We note that as expected the lower limits given by the profile likelihood analysis lie lower than the limits given by the PDF analysis. The analysis thus indicates that the light particles in mSUGRA in view of the Higgs mass measurement are the neutralino, the chargino, the gluino, the stau and the stop. Among these the most likely candidates for discovery in the next phase of CERN experiment are the 

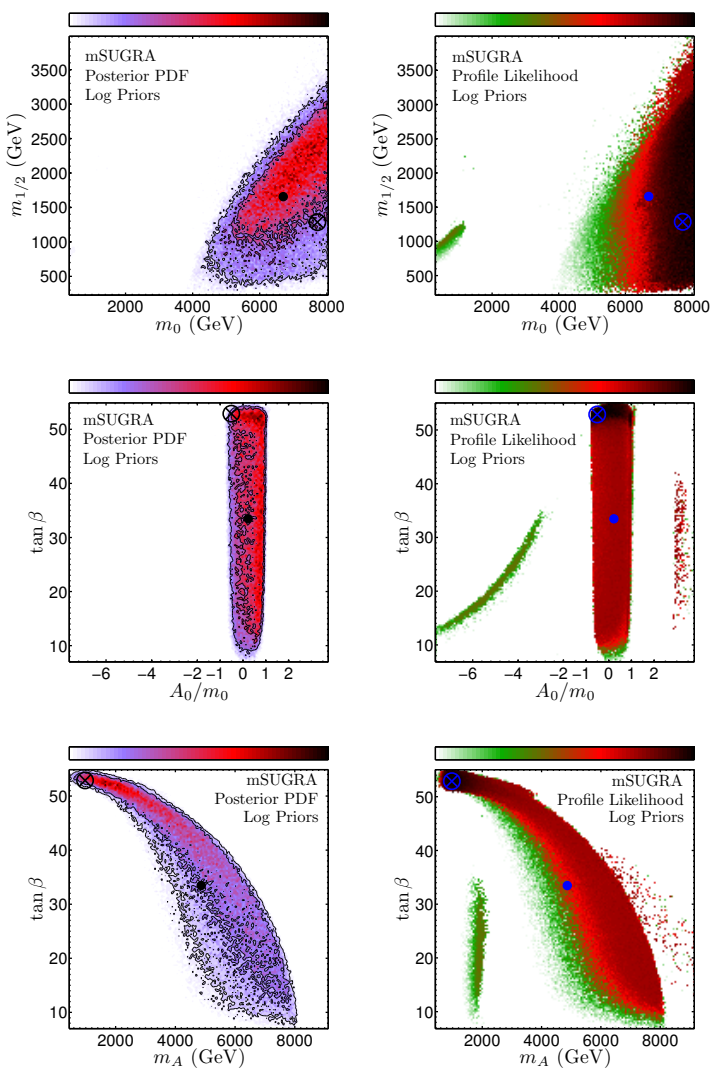

FIG. 1: Left panels: plots of the 2D posterior probability densities, $1 \sigma$ and $2 \sigma$ contours are also drawn. Right panels: plots of the profile likelihoods. Top: in the $m_{0}-m_{1 / 2}$ plane. Middle: in the $A_{0} / m_{0}-\tan \beta$ plane. Bottom: in the $m_{A}-$ $\tan \beta$ plane. The posterior mean is marked by a large dot while the best-fit point is shown by a circled ' $\mathrm{X}$ '. The color bar above the top panel gives the relative likelihood which increases left-to-right.

gluino, the chargino and the stop.

$125 \mathrm{GeV}$ Higgs boson and dark matter: Neutralinoproton spin independent cross section $\sigma_{\tilde{\chi}_{1}^{0} p}^{\mathrm{SI}}$ depends sensitively on the Higgs boson mass (for a discussion see [6]). Thus considering the $\sim 125 \mathrm{GeV}$ Higgs mass leads to a more constrained prediction for dark matter. In Fig. 3 we give a plot of $\mathcal{R} \times \sigma_{\tilde{\chi}_{1}^{0} p}^{\mathrm{SI}}$ as a function of the lightest neutralino mass $m_{\tilde{\chi}_{1}^{0}}$ where the factor $\mathcal{R}$ is defined by $\mathcal{R} \equiv\left(\Omega h^{2}\right) /\left(\Omega h^{2}\right)_{\text {WMAP }}$, and $\left(\Omega h^{2}\right)_{\text {WMAP }}$ is the central value of the WMAP-7 data. By only applying a likelihood penalty for points that are above the WMAP-7 limit, we have taken into account the possibility that there may be additional components of dark matter beyond the neutralino 30 . Quite remarkably, the bulk of the credible region of mSUGRA falls essentially exclusively between the current limits on dark matter by XENON-100 31] and the projected sensitivity of SuperCDMS [33] and XENON-1T [32.

We discuss now the constraint from $g_{\mu}-2$. In su-
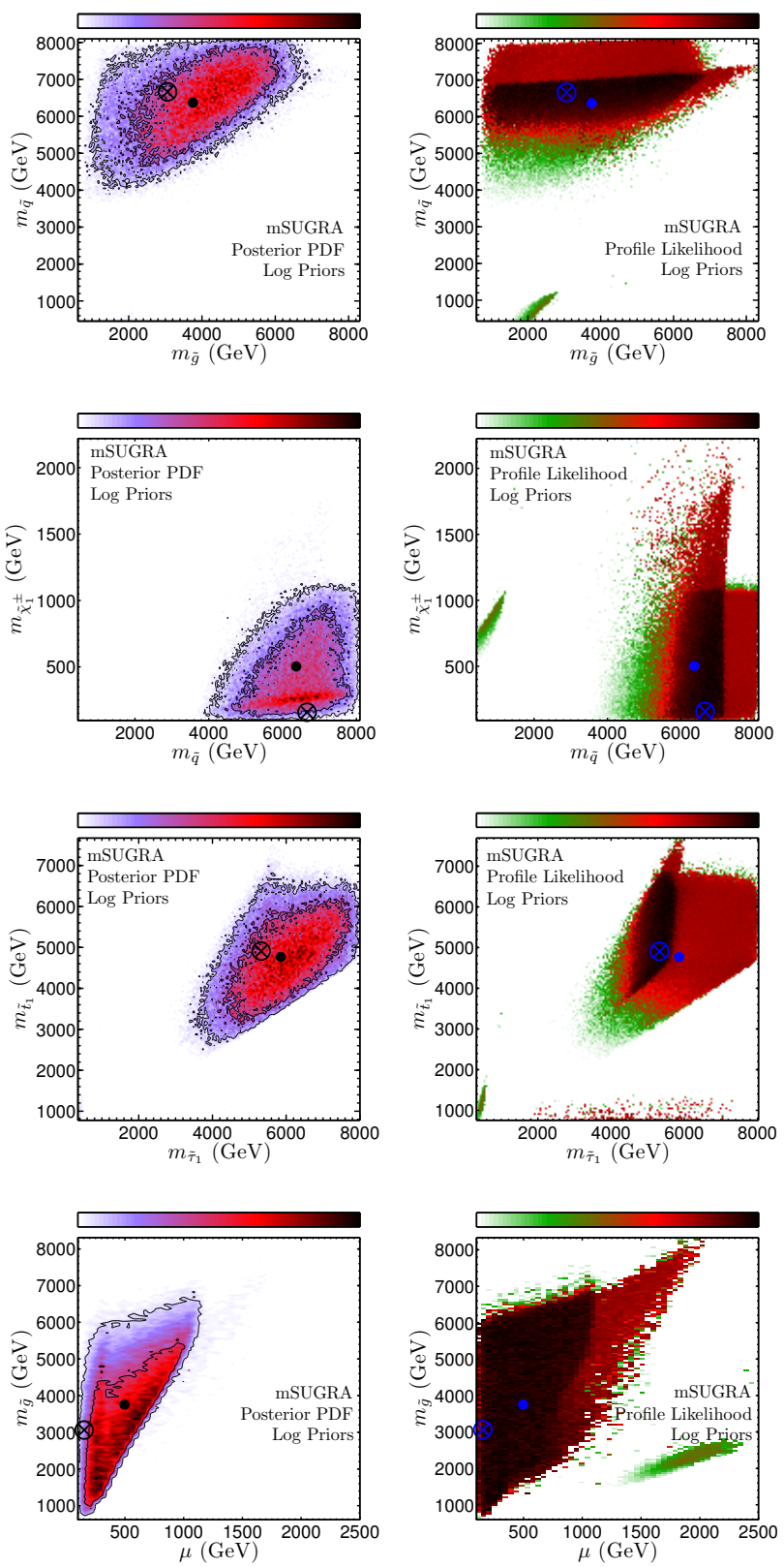

FIG. 2: Left panels: plots of the 2D posterior probability densities, $1 \sigma$ and $2 \sigma$ contours are also drawn. Right panels: plots of the profile likelihoods. Top: in the $m_{\tilde{g}}-m_{\tilde{q}}$ plane. Upper-middle: in the $m_{\tilde{q}}-m_{\tilde{\chi}_{1}^{ \pm}}$plane. Lower-middle: in the $m_{\tilde{\tau}_{1}}-m_{\tilde{t}_{1}}$ plane. Bottom: in the $\mu-m_{\tilde{g}}$ plane. The posterior mean is marked by a large dot while the best-fit point is shown by a circled ' $\mathrm{X}$ '. The color bar above the top panel gives the relative likelihood which increases left-to-right.

persymmetric theories, sparticle loops make significant contributions to the anomalous magnetic moment of the muon 29] if the relevant sparticles (charginos, neutralinos, smuons, sneutrinos) entering the loops are relatively light. The experimental determination of $\delta a_{\mu}=a_{\mu}^{\exp }-a_{\mu}^{\mathrm{SM}}$ where $a_{\mu}=\left(g_{\mu}-2\right) / 2$, de- 

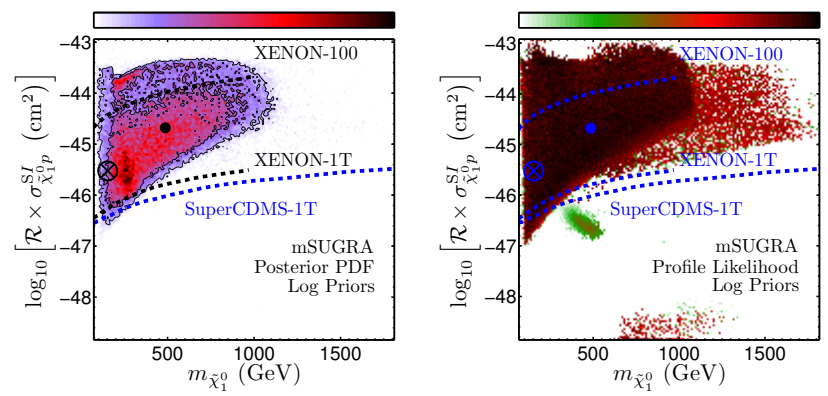

FIG. 3: (color online) Plots of $\mathcal{R} \equiv\left(\Omega h^{2}\right) /\left(\Omega h^{2}\right)_{\text {WMAP }}$ vs the neutralino mass $m_{\tilde{\chi}_{1}^{0}}$. The left panel presents the $2 \mathrm{D}$ posterior PDF, and the right panel presents the profile likelihood. The analysis shows that virtually all of credible region of mSUGRA will be probed by the SuperCDMS 33] and XENON-1T [32] experiments. The color bar above the panels gives the relative likelihood which increases left-to-right.

pends sensitively on the hadronic correction to the standard model value. There are two main procedures for the estimation of the hadronic correction, which are either using the $e^{+} e^{-}$annihilation cross section or from $\tau$ decay. The result using the $e^{+} e^{-}$ annihilation gives $\delta a_{\mu}=(28.7 \pm 8.0) \times 10^{-10}(3.6 \sigma)$ while for $\tau$-based hadronic contributions one has $\delta a_{\mu}=(19.5 \pm 8.3) \times 10^{-10}(2.4 \sigma)$ [34. In any case, within the universal soft SUSY-breaking paradigm there would be tension between the $g_{\mu}-2$ result (specifically the one using $e^{+} e^{-}$annihilation cross section) and the $125 \mathrm{GeV}$ Higgs boson mass since the $m_{0}$ scale is rather high. If the $g_{\mu}-2$ results stay, then there are at least two avenues open to make compatible the $g_{\mu}-2$ results and the Higgs boson mass. The first possibility is that we stay within the universal soft breaking paradigm and additional contributions to the Higgs mass arise due to the presence of extra matter which can generate new loop corrections to the Higgs mass, or from extra gauge groups under which the Higgs is charged yielding corrections to the Higgs mass through $D$-terms. Alternatively, one could give up universality of soft parameters and consider non-universal or flavored SUGRA models 35. For instance, to satisfy the $g_{\mu}-2$ constraint one may consider the soft scalar mass for the first two generations much smaller than for the third generation, or the sleptons being lighter than the squarks. These possibilities require further investigation.

Conclusion: In this work we have analyzed the implications of the Higgs boson discovery at CERN for supersymmetry. Specifically we analyzed the mSUGRA model to delineate constraints on soft parameters and identified the light particles that are prime candidates for discovery in the next phase of runs at the LHC. The analysis presented here explains why supersymmetric dark matter has not been seen thus far since essentially all of the pa- rameter space lies below the current sensitivity of dark matter experiments due to the high Higgs mass. The analysis also points to excellent prospects for the discovery of dark matter at SuperCDMS and XENON-1T as well as the possibility of light neutralinos, charginos and gluinos, and possibly light stops and staus.

One of us (S.A.) thanks TASI-2012 where part of this work was done. This research is supported in part by NSF Grants Nos. PHY-0757959, and PHY-0969739, and used resources of the National Energy Research Scientific Computing Center, which is supported by the Office of Science of the U.S. Department of Energy under Contract No. DE-AC02-05CH11231.

[1] J. Incandela [on behalf of CMS], "Status of the CMS SM Higgs Search", and F. Gianotti [on behalf of ATLAS], "Status of the SM Higgs Search in ATLAS", joint CMS/ATLAS seminars at CERN, July 4, 2012; CMS Collaboration, CMS-PAS-HIG-12-020; ATLAS Collaboration, ATLAS-CONF-2012-093.

[2] S. Chatrchyan et al. [CMS Collaboration], Phys. Lett. B arXiv:1207.7235 [hep-ex]]; G. Aad et al. [ATLAS Collaboration], Phys. Lett. B arXiv:1207.7214 [hep-ex]].

[3] F. Englert and R. Brout, Phys. Rev. Lett. 13, 321 (1964); P. W. Higgs, Phys. Lett. 12, 132 (1964). Phys. Rev. Lett. 13, 508 (1964); G. S. Guralnik, C. R. Hagen and T. W. B. Kibble, Phys. Rev. Lett. 13, 585 (1964).

[4] A. H. Chamseddine, R. L. Arnowitt, P. Nath, Phys. Rev. Lett. 49, 970 (1982); P. Nath, R. L. Arnowitt, A. H. Chamseddine, Nucl. Phys. B227, 121 (1983); L. J. Hall, J. D. Lykken, S. Weinberg, Phys. Rev. D27, 2359-2378 (1983); R. L. Arnowitt and P. Nath, Phys. Rev. Lett. 69, 725 (1992); P. Nath, hep-ph/0307123.

[5] L. E. Ibanez and G. G. Ross, Comptes Rendus Physique 8, 1013 (2007).

[6] S. Akula et al., Phys. Rev. D 85, 075001 (2012).

[7] A. Arbey et al., arXiv:1207.1348 [hep-ph].

[8] Y. Okada et al., Prog. Theor. Phys. 85, 1 (1991); J. R. Ellis et al., Phys. Lett. B 257, 83 (1991); H. E. Haber and R. Hempfling, Phys. Rev. Lett. 66, 1815 (1991); J. A. Casas et al., Nucl. Phys. B 436, 3 (1995).

[9] A. Djouadi, Phys. Rept. 459, 1 (2008); M. S. Carena and H. E. Haber, Prog. Part. Nucl. Phys. 50, 63 (2003)

[10] [CMS Collaboration], Phys. Lett. B 710, 26 (2012); [ATLAS Collaboration], arXiv:1207.0319 [hep-ex].

[11] [The TeVatron], arXiv:1207.0449 [hep-ex].

[12] H. Baer et al., Phys. Rev. D 85, 075010 (2012); A. Arbey et al., Phys. Lett. B 708, 162 (2012); J. L. Feng et al., Phys. Rev. D 85, 075007 (2012); M. Carena et al., JHEP 1203, 014 (2012); S. Heinemeyer et al., Phys. Lett. B 710, 201 (2012); P. Draper et al., Phys. Rev. D 85, 095007 (2012); J. Ellis and K. A. Olive, Eur. Phys. J. C 72, 2005 (2012); O. Buchmueller et al., arXiv:1112.3564 [hep-ph]; U. Ellwanger, JHEP 1203, 044 (2012).

[13] M. Kadastik et al., JHEP 1205, 061 (2012); J. Cao et al., Phys. Lett. B 710, 665 (2012); B. Batell et al., arXiv:1112.5180 [hep-ph]; L. J. Hall et al., JHEP 1204, 131 (2012); J. F. Gunion et al., Phys. Lett. B 710, 454 (2012); P. Fileviez Perez, Phys. Lett. B 711, 
353 (2012); S. F. King et al., Nucl. Phys. B 860, 207 (2012); J. Cao et al., JHEP 1203, 086 (2012); C. F. Chang et al., JHEP 1206, 128 (2012); L. Aparicio et al., JHEP 1204, 126 (2012); H. Baer et al., JHEP 1205, 091 (2012); D. Ghosh et al., arXiv:1202.4937 [hep-ph]. N. Desai et al., arXiv:1202.5190 [hep-ph]; D. Carmi et al., arXiv:1202.3144 [hep-ph]; L. Maiani et al., New J. Phys. 14, 073029 (2012); T. Cheng et al., arXiv:1202.6088 [hep-ph]; J. Cao et al., JHEP 1206, 145 (2012); F. Jegerlehner, arXiv:1203.0806 [hepph]; N. D. Christensen et al., arXiv:1203.3207 [hep-ph]; A. Choudhury and A. Datta, JHEP 1206, 006 (2012); B. S. Acharya et al., Int. J. Mod. Phys. A 27, 1230012 (2012); M. A. Ajaib et al., arXiv:1204.2856 [hep-ph]; J. Ellis and T. You, arXiv:1204.0464 [hep-ph]; C. Balazs et al., arXiv:1205.1568 [hep-ph]; J. L. Feng and D. Sanford, arXiv:1205.2372 [hep-ph]; C. Boehm et al., arXiv:1205.2815 [hep-ph]; N. Okada, arXiv:1205.5826 [hep-ph]; M. Carena et al., arXiv:1205.5842 [hep-ph]; E. Dudas et al., arXiv:1205.5988 [hep-ph]; A. Fowlie et al., arXiv:1206.0264 [hep-ph]; M. Hirsch et al., arXiv:1206.3516 [hep-ph]; R. M. Chatterjee et al., arXiv:1206.5770 [hep-ph]; M. W. Cahill-Rowley et al., arXiv:1206.5800 [hep-ph]; A. Albaid and K. S. Babu, arXiv:1207.1014 [hep-ph].

[14] J. Beringer et al., Phys. Rev. D 86 (2012) 010001.

[15] E. Komatsu et al., Astrophys. J. Suppl. 192, 18 (2011).

[16] E. Barberio et al. arXiv:0808.1297[hep-ex]; M. Misiak et al., Phys. Rev. Lett. 98, 022002 (2007).

[17] R. Aaij et al.,Phys. Rev. Lett. 108, 231801 (2012).

[18] F. Feroz et al., Mon. Not. Roy. Astron. Soc. 398, 1601 (2009); F. Feroz et al., JHEP 1106, 042 (2011).

[19] S. Akula, SusyKiT. http://freeboson.org/software/.

[20] B. C. Allanach, Comput. Phys. Commun. 143, 305
(2002)

[21] G. Belanger et al., Comput. Phys. Commun. 180, 747 (2009); Comput. Phys. Commun. 182, 842 (2011).

[22] L. Roszkowski et al., arXiv:0707.0622 [astro-ph]; JHEP 0707 (2007) 075; JHEP 0704 (2007) 084; R. R. de Austri et al., JHEP 0605 (2006) 002; F. Feroz et al., arXiv:0809.3437 [astro-ph]; R. Trotta et al., New Astron. Rev. 51 (2007) 316; JHEP 0812, 024 (2008); JCAP 0908 (2009) 034.

[23] A. Lewis and S. Bridle, Phys. Rev. D 66, 103511 (2002).

[24] Y. Akrami et al., JHEP 1004, 057 (2010).

[25] S. Akula et al., Phys. Lett. B 709, 192 (2012).

[26] K. L. Chan et al., Phys. Rev. D 58 (1998) 096004; U. Chattopadhyay et al., Phys. Rev. D 68, 035005 (2003). H. Baer et al., JHEP 0306, 054 (2003).

[27] J. L. Feng et al., Phys. Rev. Lett. 84, 2322 (2000).

[28] D. Feldman et al., arXiv:1105.3765 [hep-ph].

[29] T. C. Yuan et al., Z. Phys. C 26, 407 (1984); D. A. Kosower et al., Phys. Lett. B 133, 305 (1983).

[30] D. Feldman et al., Phys. Rev. D 81, 095017 (2010); W. Z. Feng et al., Phys. Rev. D 85, 115016 (2012).

[31] E. Aprile et al. [XENON100 Collaboration], arXiv:1207.5988 [astro-ph.CO]; E. Aprile et al. [XENON100 Collaboration], Phys. Rev. Lett. 107, 131302 (2011); E. Aprile et al. [XENON100 Collaboration], Phys. Rev. D 84, 052003 (2011).

[32] E. Aprile, The XENON Dark Matter Search, WONDER Workshop, LNGS, March 22, 2010.

[33] B. Cabrera, "SuperCDMS Development Project", 2005.

[34] A. Hoecker, Nucl. Phys. Proc. Suppl. 218, 189 (2011); K. Hagiwara et al., J. Phys. G G 38, 085003 (2011).

[35] P. Nath and R. L. Arnowitt, Phys. Rev. D 56, 2820 (1997). 